\title{
Balsalazide Disodium
}

National Cancer Institute

\section{Source}

National Cancer Institute. Balsalazide Disodium. NCI Thesaurus. Code C47409.

The disodium salt form of balsalazide, an aminosalicylate and oral prodrug that is enzymatically cleaved in the colon to produce the anti-inflammatory agent mesalazine. Mesalazine acts locally on the mucosa of the colon where it diminishes inflammation by blocking the production of arachidonic acid metabolites, including leukotrienes, prostaglandins and hydroxyeicosatetraenoic acids, and other inflammatory agents. Balsalazide disodium is used to treat chronic inflammatory bowel disease. 